Session 2320

\title{
InFusion: Simplifying Online Course Creation
}

\author{
Joel R. Jackson, Thomas P. Barnwell III, \\ David V. Anderson, Monson H. Hayes III
}

\begin{abstract}
In order for faculty to become involved in the development of online or computer enhanced lecture materials, the production process must be easy and convenient. We have developed a presentation development system called inFusion that makes it possible to create online presentations almost instantly. Lecturers use a very simple interface (below, right) to synchronize graphics, video and audio into a coherent presentation viewable in any browser, as shown below.
\end{abstract}

These lecture modules consist of multiple synchronized media types. A typical application is synchronization of still slides with a talking head. However, media can also include animations, audio, multiple video clips, multiple synchronized still images, and text. The presentation format is flexible, allowing the presenter to change backgrounds, the elements included, and the layout. This flexibility allows an instructor to tailor presentations to his teaching style, the learning style of his students, and the material. Any of the media windows can be made "hot," with links to supplementary material, readings, or other lectures. These links can change as the presentation progresses, allowing the creation of context-sensitive links to additional material.

The primary advantages of inFusion are simplicity and portability. Although many of the Internet courses we have produced at Georgia Tech have been filmed in a small studio, instructors can also capture lectures in the classroom, in their own office, or at home. A portable production system can consist of as little as a laptop and a USB camera. Instructors, even with little or no previous experience with inFusion, can create effective and engaging online lectures without the need for additional production personnel. We have found that the availability of these tools has increased interest in creating online courses among the faculty at Georgia Tech.

We have made inFusion freely available at www.ece.gatech.edu/streaming.

\section{Introduction}

Georgia Tech is involved in a number of distance learning initiatives, including a new online Masters program in Electrical and Computer Engineering and a campus situated in Metz, France, that also offers Masters degrees in Electrical and Computer Engineering. This campus, known as 
Georgia Tech Lorraine (GTL), opened its doors to students in the Fall of 1991. Since the number of faculty at GTL has never been large enough to support a complete Masters Degree program, a number of courses are typically offered through the Georgia Tech Center for Distance Learning. These courses, which are taught by Georgia Tech faculty to students in Atlanta, are videotaped, and the tapes are shipped once a week to GTL. There are many problems associated with the offering of courses by videotape. For example, shipping videotapes across the Atlantic is costly. In addition to the weekly express mail charges, there is the time and expense involved in duplicating tapes, and the cost of packaging and mailing approximately 45 videotapes per course back to Atlanta at the end of each semester. A more serious set of problems is that associated with the unavoidable time delays that result from shipping videotapes, and these delays create problems for both students and faculty. At a minimum, this delay is a week, since each Friday the tapes for that week are mailed to GTL. Another problem with videotapes is the lack of interaction between faculty and student - the student is not able to stop the instructor to ask questions. Finally, it is generally acknowledged that videotaped lectures tend to be dull and uninteresting since all that is typically recorded is the instructor writing on a board or lecturing from overhead slides.

Because of these problems, in the summer of 1997 we delivered a course from GTL to Atlanta using the Internet ${ }^{1,2}$. This course consisted of PowerPoint slides that were synchronized to streaming audio of the lecture. Due to the efficiency of this method, the near instantaneous access students had to course materials, and the enthusiastic support of our distance learning students, we began looking at more effective ways to use the Internet to deliver courses, and today we are streaming audio, video, text, and slides. In some lectures, the video consists of chromakey video. And yet, in spite of the advantages that the Internet offers in course delivery, there remain obstacles to its widespread acceptance.

\section{Course production tools}

There are many advantages to offering courses over the Internet instead of using more traditional methods, such as videotapes, and there is no question that Internet distance learning will become widespread in the future ${ }^{3,4}$. In fact, today we are currently witnessing an explosion in the delivery of on-line courses ${ }^{5}$, and some universities are putting complete degree programs on the Web. The Internet offers the ability to deliver courses throughout the world, any time and any place. Internet-based courses also offer the student fast and efficient navigation through a lecture, fast retrieval of multimedia content, and course and lecture indexing. Internet courses may also offer keyword searches through multimedia content, self-assessment tools, interactive content, and hyperlinks to related course material.

In spite of these advantages, there are some problems and pitfalls in offering courses over the Internet. Some of these will disappear in the future, while others will continue to present difficulties for the foreseeable future. We have found at Georgia Tech that, in spite of seminars that we have given to faculty to encourage them to get involved with Internet distance learning, faculty participation is spotty at best, although faculty curiosity and interest is high. Other studies indicate that the faculty at Georgia Tech is not alone regarding their reluctance to participate in 
distance education ${ }^{6}$. There are many reasons for this. First, to develop a course for Internet delivery, it is necessary for faculty to commit a significant amount of time creating the course content. Unfortunately, there may never be a way to ameliorate this, short of hiring a staff of content design experts. Secondly, there are constraints that are placed on faculty when teaching courses for delivery over the Internet. Since some teaching styles do not lend themselves to Internet delivery, such as writing on a white board, faculty must be willing to adapt the way that they teach. Another aspect of distance learning that some instructors will find to be a serious drawback is the lack of contact with students, and the enlarged role that faculty must assume in encouraging and facilitating interaction with the students, which is unnecessary in traditional settings. Finally, there is a significant investment in start-up time required to design a course and bring it on-line. This is due to the absence of powerful and effective tools that enable the instructor to design and develop course content and bring it on-line seamlessly. To makes things worse, faculty investing the time and effort to produce Internet-based courses typically do not receive any extra compensation or release time.

Recognizing the existence of these problems, in the fall of 1998 we began looking at ways to facilitate the development of on-line courses. What was clear was that, once the course content was created, in order to produce a streaming media course, a tremendous amount of tedious work is required. This includes the extraction of timing information for slide synchronization, the writing of SMIL files that orchestrate the streaming media production, and the design of the HTML pages and links. Therefore, our first task was to write a scripting program that would automate the recording of timing information and generate all of the necessary streaming media files. The result was a set of simple tools, written in Perl, that reduced the production time down from a couple of hours to a few minutes. The next step was then to develop a user-friendly user front-end that would allow an instructor to publish his or her content for Internet delivery without having to use command line instructions. At the same time, some useful generalizations and extensions to the original program were added. The end result is a PC program for producing course "modules" that is powerful, yet easy to use. The user interface is illustrated in Figure 1.

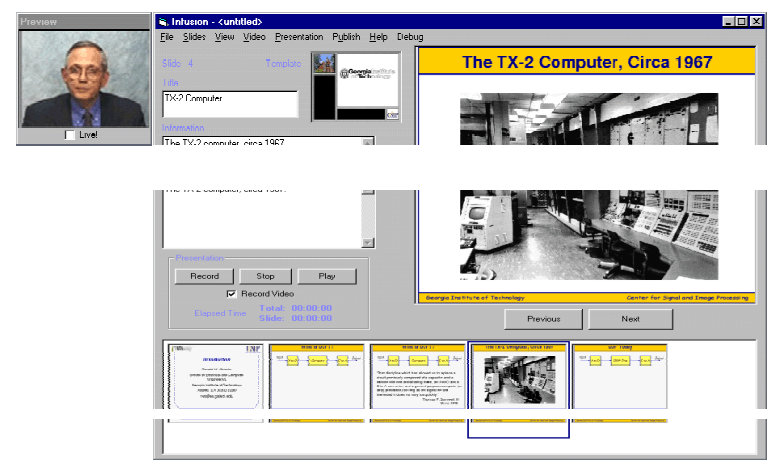

Fig. 1. The user interface.

The instructor needs to provide only postscript files containing the slides or individual images of the slides in GIF, JPEG or PNG format. Then, with a video camera focused on the instructor, a 
lecture module that includes synchronized video, audio, images, outline and mouse clicks (for annotation use) is captured to disk. Once the video has been encoded, the tool is then ready to automatically generate all of the necessary streaming media files. An example illustrating the presentation format these files generate is shown in Figure 2. The presentation format is flexible, allowing the user to change backgrounds, the elements included, and the layout. This allows the creation of lectures including only audio, or with additional information in the windows around the slide, thus allowing the instructor to tailor the presentation to his teaching style or the learning style of his students. Any of the media windows can be made "hot-clickable," with links to supplementary material that change as the presentation progresses. This allows a single window to be used as a "Supplementary Information" window, with extra readings or links that correspond to the portion of the lecture currently being viewed. The primary advantages of these tools are simplicity and portability. Although many of our Internet courses are filmed in a small studio, instructors can capture lectures in their own office, at home, or in the classroom.

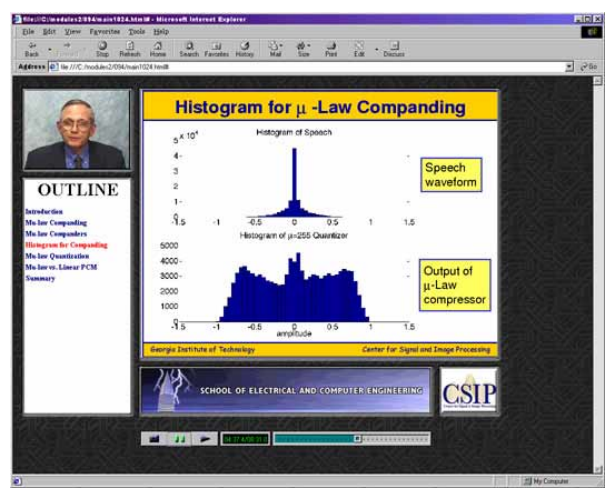

Fig. 2. Screen shot of a presentation produced using inFusion $($.

There are two approaches that may be used for online course presentation. The first is to "capture" the classroom during a live lecture, and place it on the Internet for distance learning students. The second is to produce lectures or lecture modules outside of class in a studio or office, and produce an Internet version of a course. In the second case, our tools allow simple capture and placement on the Internet. In addition, the unique elements of internet-based education such as increased interactivity and hierarchical arrangement of information are easy to implement. In the classroom, the tools would be used to capture the events occurring during the lecture, possibly including whiteboard notes, and all of the timing information for synchronization. The resulting presentation could be used as is or modified to match the particular teaching style of the instructor. Construction of additional multimedia files could be done outside of class at a later time.

With a simple set of tools to bring synchronized and indexed audio easily onto the Internet, the next set of issues that should be addressed center around how to make effective course content that will enhance the learning environment for the student.

\section{Course content}


Developing distance learning environments offers the opportunity to redefine the classroom concept, and to expand the set of tools available to the instructor. These tools can be grouped into two categories: lecture delivery and enhanced materials. The first provides the applications necessary to produce and deliver the fundamental lecture from the primary site. The latter category deals with those materials that enhance the learning environment and expand the resources available to the student. Over the last few years, we have given a lot of thought on how to enhance the learning environment, and we are now beginning to implement some of these enhancements.

The first enhancement that we are in the process of developing involves the development of effective ways that will allow the student to efficiently search for information within the course content. For example, we believe that the student should be able to enter key-words into a computer, either by typing them using the keyboard or by speaking into a microphone, to find some desired information or content. More generally, the student may wish to search for information about a topic over an entire library of courses. Although, eventually, the student should be able to search over all types of media, i.e., text, graphics, audio, and video, up to this point in time we have only focused on searching over textual information, such as slides. Clearly, the generation of the index that is to be used for content searching should be automated so that key-words do not need to be entered manually into a database, or manually linked to audio and video content. Therefore, what we are developing is a program automatically generating a keyword index from postscript slides, and linking these words back to the multimedia course content.

The communication requirements inherent in the teacher-student relationship are another obstacle that must be overcome in a remote learning environment [8]. In some courses, we are currently using traditional teleconferencing via PictureTel systems for group sessions. We have also used Internet based video conferencing for online office hours between students and teachers. We are expanding the use of the Internet based solution for cost reasons, and availability to the student population. Several packages are being evaluated for use in the production environment; Microsoft NetMeeting and Netscape Conference are primary candidates.

Another enhancement that we believe will be important in the future is to allow the student to be able to interact with his/her learning environment. This will involve a number of different aspects. First, the student should be provided with an effective note-taking environment. This would include allowing the student to be able to capture text and graphics information for annotation. In addition, the student may wish to caption short audio or video clips for future listening or viewing. The student should also be given the tools that would enable him/her to apply electronic post-it notes to tag information that is important or confusing. For information that is confusion, the student should be able to easily send the content along with the post-it note to the instructor for clarification.

Assessment of student learning is, without a doubt, something that will be important in any electronic course. For example, it will be important for the student to be able to assess his or her understanding of a course module before proceeding onto the next. There are several ways that this 
assessment could be performed, and which one is best is a subject for debate. One approach would be to have a set of questions, some percentage of which must be answered correctly, before he/she is allowed to proceed onto the next module. Alternatively, the student could be directed to some other course material or problems to work if some deficiency in understanding is detected.

Finally, as course development tools and software become more sophisticated and readily available, we may find the student being able to proceed through the course using a path that depends on his/her learning styles. We expect, for example, learning theory to become increasingly important in developing Internet-based courses in the future.

Over the next few years, we will begin to see some new technologies being used in courses that are delivered over the Internet. These new technologies will undoubtedly include the following: Tools that will automatically search and index audio using voice recognition technologies, The incorporation of natural language interfaces for information searching and retrieval, Synthesis of speech from text to facilitate learning for the visually impaired, and automatic captioning for the hearing impaired.

However, perhaps the most important change that the next century will bring is the availability of production tools that will enable faculty to develop and deliver courses easily across the Internet. Today, the process is extremely expensive and time-consuming. As a result, Internet education is not yet widespread. We believe that Internet distance learning will continue to gain momentum and, given the need for production tools, they will begin to appear on the marketplace.

\section{Conclusions}

We believe that the Internet will be used extensively in the future to deliver educational material to remote students or distant educational institutions. In addition, we expect that the corporate environment will rely more heavily on the Internet in the future for delivering training materials or corporate information to their employees. As a result, there will be a heavy demand for simple and effective content production and delivery tools. In addition, there will be a lot of discussions on how to most effectively train and educate the "Internet Student." In this paper, we have described some of our efforts in developing effective tools for publishing Internet-based courses, and we have outlined some of the things that we believe will be necessary and important for an effective learning environment for the student.

\section{Bibliography}

1. M.H. Hayes, "Some approaches to Internet distance learning with streaming media", IEEE Second Workshop on Multimedia Signal Processing, pp. 514-519, Los Angeles, CA, Dec. 1998.

2. M.H. Hayes and L.D. Harvel, "Distance learning into the 21st century", Proc. ASEE Workshop, Charlotte, NC, June 1999.

3. J.R. Bourne, A.F. Mayadas and J.O. Campbell, "Asynchronous learning networks: an information-technology-based infrastructure for engineering education”, Proc. IEEE, vol. 88, pp. 63-71, Jan. 2000. 
4. A. P. Sanoff, "Long-distance relationship", ASEE Prism, pp. 22-26, Sept. 1999.

5. P. Brusilovsky, "Web lectures: electronic presentations in web-based instruction", Syllabus, pp. 18-23, Jan. 2000.

6. K. Betts, "Why do faculty participate in distance education?", Horizon Web Magazine, Oct. 1998.

7. M.H. Hayes and M. Jamrozik, "Internet distance learning - The problems, the pitfalls, and the future", Proceedings of IEEE Workshop on Multimedia Signal Processing, pp. 569-574, Copenhagen, Denmark, Sept. 1999.

8. C. Williamson, J.T. Bernhard and K. Chamberlin, "Perspectives on an Internet-based synchronous distance learning experience", J. Engineering Education, vol. 89, pp. 53-61, Jan. 2000.

\section{JOEL R. JACKSON}

Dr. Joel Jackson is currently an assistant professor of Computer Engineering with the Georgia Tech Regional Engineering Program at Georgia Tech. He has been involved in developing methods for computer-enhanced education and distance learning in the Center for Signal and Image Processing. His research includes DSP with applications in medical imaging and remote sensing, DSP education, and embedded medical imaging devices.

\section{THOMAS P. BARNWELL}

Dr. Thomas P. Barnwell III received his B.S. degree in 1965, his M.S. degree in 1967, and his Ph.D. degree in 1970, from M.I.T. He has been principal investigator on numerous research contracts and grants in the areas of speech coding and analysis, objective quality measures for speech, multiprocessor architectures for digital signal processing, and computer networking and distributed processing.

\section{MONSON H. HAYES III}

Dr. Monson H. Hayes received the Sc.D. degree in Electrical Engineering from M. I. T in 1981. He was an Associate Editor in signal processing for the IEEE Transactions on Acoustics, Speech, and Signal Processing from 1984 to 1986, and Secretary-Treasurer of the ASSP Publications Board. He is the recipient of the 1983 IEEE Senior Award for the author of a paper of exceptional merit and is the recipient of a 1984 Presidential Young Investigator Award.

\section{DAVID V. ANDERSON}

Dr. David Anderson. He received his B.S. and M.S. from Brigham Young University, and his Ph.D. from Georgia Institute of Technology in 1999. David's research interests include audition and psycho-acoustics, signal processing in the context of human auditory characteristics. He is actively involved in the development and promotion of computer enhanced education. He is a member of the IEEE, the Acoustical Society of America, and the Society for Industrial and Applied Mathematics. 